\title{
Nostos y Laberinto
}

\author{
Eduard Vilella
}

Universitat Autònoma de Barcelona

\section{Resumen}

Las múltiples referencias en la obra de Vincenzo Consolo al tema clásico del viaje de regreso y en especial al de Ulises pueden ser consideradas como un auténtico leitmotiv. En la cita reiterada al nostos homérico se puede observar como el autor siciliano elabora una constelación de preocupaciones que se revela fundamental en su actividad narrativa y ensayística. Confluyen en este nucleo haces provenientes de un amplio espectro extendido entre lo más explícitamente político o ético-moral y la reflexión más íntima acerca del objeto artístico, pasando por aspectos centrales en Consolo como los de tipo existencial, la inquietud por el territorio o los relacionados con la destrucción estilística y formal de la novela tradicional, entre otros. El artículo parte de la descripción de esta realidad, y los análisis críticos que han discurrido sobre ella, para intentar trazar un paralelismo entre ésta y el uso del tema del laberinto en Consolo, tomando como foco principal de atención el episodio de la cárcel-espiral que aparece en la parte final de Il sorriso dell'ignoto marinaio.

Palabras clave: narrativa, ensayo; nostos; laberinto; ética de la escritura consoliana; literatura y territorio sicilianos.

\section{Abstract}

The numerous references in the work of Vincenzo Consolo to the classic notion of the homeward journey, in particular that of Ulysses, could be considered an authentic leitmotiv. Through his repeated references to Homer's nostos, one can see how the Sicilian author develops a myriad of concerns that turn out to be essential to his storytelling and essays. Within this nucleus we can find beams flooding in from a broad spectrum, spanning that which is most assertively political or ethical/moral, as well as the most intimate reflections on the object of art, whilst also encompassing core aspects of Consolo's work, such as existentialism, concerns over land or those related to the formal and stylistic destruction of the traditional novel, amongst other things. The article starts out with a description of this reality, together with the critical analyses it has attracted, the aim being the attempt to trace a parallel between such reality and Consolo's use of the labyrinth theme, taking the episode of the prison-spiral, which appears in the final part of Il sorriso dell'ignoto marinaio (The Smile of the Unknown Mariner), as the main focus of attention.

Key words: Vincenzo Consolo: storytelling, essay; nostos; labyrinth; ethics of Consolo's writing; Sicilian literature and land. 
Es bien conocida la huella dejada por los textos homéricos en la obra de Vincenzo Consolo, particularmente explícita en sus últimos libros. Para ser más precisos, es sobre todo de la esfera de los nostoi, los viajes de regreso, que hay que hablar y, en concreto, como cabría esperarse, del más ilustre de estos viajes, es decir, el de Ulises. Intertexto constante en una más que notable serie de textos del autor siciliano, la Odisea efectivamente adquiere en ocasiones la silueta de un auténtico hipotexto, fundamento y referencia ineludible para una lectura de Consolo. Cabrá referirse en primer lugar a lo que la crítica ha llamado las novelas de Odiseo (L'Olivo e l'olivastro, Lo Spasimo di Palermo) acerca de cuya referencia a la Odisea, manifiesta por otra parte, el autor se ha extendido explícitamente en entrevistas y ensayos. ${ }^{1}$ Pero no habrá que olvidar por ello la insistencia con que Consolo, en el marco de toda su obra, vuelve al argumento, sea en la ficción sea en pasajes de tipo más discursivo (artículos, ensayos, en el libro coloquio, no cabe decir, dedicado al viaje de Ulises), ${ }^{2}$ para redundar en un reconocimiento abierto cuya sombra podría incidir incluso en narraciones escritas anteriormente, como Nottetempo casa per casa, Le Pietre di Pantalica o Il sorriso dell'ignoto marinaio. Se trata de una realidad que bien podríamos describir como leitmotiv, donde convergen diferentes planos de significación. Aunque el riesgo de la obviedad es indiscutible, puede tener su interés acercarse a la cuestión en lo que tiene de reflejo de la global estrategia artística de Vincenzo Consolo, su actitud ante el texto narrativo, la carga ideológica que se le atribuye, las decisiones estilísticas en que estos aspectos se materializan, la concepción militante del texto literario - ámbitos todos ellos que sólo, es bien sabido, en una mirada apresurada podrían parecer articularse de forma disonante- ${ }^{3}$ Aun así, podría no desaparecer la impresión de mera evidencia al

1. Por lo que se refiere a una panorámica globalmente descriptiva, entre los numerosos textos que podrían citarse, cfr. Giuseppe Traina, Vincenzo Consolo, Fiesole: Cadmo, 2001, p. 40 s. (para una descripción completa de la temática en cuestión, con numerosas indicaciones y reflexiones de interés); Enzo PAPA, «Vincenzo Consolo», Belfagor, 58:2, 2003, p. 179198, en especial p. 189 s.; Fabio Gambaro, «Vincenzo Consolo. La Sicilie entre utopie et désillusion» [entrevista], Magazine littéraire, 393, 2000, p. 98-103. Abiertamente centrado en la perspectiva que nos ocupa, con la atención dirigida en particular a Lo spasimo di Palermo, la tradición del nostos y las implicaciones paralelas en referencia a los universos de la oralidad y de la escritura en el texto homérico, la tradición europea y el texto consoliano, el artículo de Giorgio GuZzETTA, «Ulisse narratore: Consolo, Pazzi, Malerba e il "racconto marino"», en Bart VAN DEN BOSSCHE y otros (a cargo de) «...E c’è di mezzo il mare». Civiltà italiana 2, Florencia: Cesati, 2002, p. 279-289.

2. Vincenzo Consolo, Mario Nicolao, Il viaggio di Odisseo, Milán: Bompiani, 1999.

3. A fin de cuentas, caras de una misma moneda; una forma de superar aquella realidad por la cual, en palabras del autor en Il sorriso dell'ignoto marinaio la historia es "Una scrittura continua di privilegiati» (p. 88, cito de Vincenzo CONSOlO, Il sorriso dell'ignoto marinaio, Milán: Mondadori, 1987 [1a ed. Turín: Einaudi, 1976]), es decir, dar la palabra a otras voces fuera del monopolio del discurso del poder. Para el desarrollo concreto de estas ideas, cabrá subrayar en primer lugar sin duda el trabajo de Cesare SEGRE, «La costruzione a chiocciola nel Sorriso dell'ignoto marinaio di Consolo", en Intrecci di voci. La polifonia nella letteratura del Novecento, Turín: Einaudi, 1991, p. 71-86 (antes en Vincenzo Consolo, Il sorriso dell'ignoto marinaio, op. cit. p. V-XVIII). Línea interpretativa que fácilmente podría hacerse extensible a toda la obra de Consolo. Es recurrente en la crítica referirse en parale- 
tratar la cuestión del nostos en Consolo; la intención de estas páginas es abordar más bien la presencia, más esporádica en el autor, del tema del laberinto, y hacerlo no aisladamente, sino en concomitancia con el tema del viaje de regreso, con el tratamiento del cual se establecen interesantes afinidades. En otras palabras, el laberinto, tal como se muestra en ciertos pasajes del autor, podría responder a coordenadas semejantes a las del nostos, con lo que uno y otro tema podrían iluminarse mutuamente.

Por lo que se refiere al primero de los temas, el nostos, como se decía, son muchas las citas que sería posible aportar. Nos detendremos sólo en alguno de los pasajes más emblemáticos para una visión panorámica de las dinámicas que emergen en su tratamiento. Tomemos como punto de partida, por una parte, las evidencias más inmediatas: el mismo autor, al abordar e intentar ofrecer un porqué a su preferencia por el esquema en cuestión, al remitirse de esta manera a una tradición en la que afirma reconocerse, define la Odisea no sólo como el texto clásico por excelencia, sino también como una verdadera estructura básica, cuyos ecos reverberan a lo largo de toda la historia de la literatura universal, en lo arquetípico de su tensión, movimiento y errancia:

$\mathrm{Ci}$ interessa chiarire che da questo grande archetipo che è l'Odissea, da questo primo racconto di viaggio, siano poi germinate infinite odissee, che quello è diventato un viaggio interminabile, che dall'immaginario greco e poi latino, mediterraneo, è passato a quello mondiale, o quanto meno a quello della nostra civiltà, della nostra letteratura occidentale. ${ }^{4}$

Se entiende así, por ejemplo, que, hablando del famoso retrato que da nombre a su novela Il sorriso..., pueda el autor conjuntar tradición secular, obra particular, valor de Ulises como epítome de la condición humana:

lo a la tensión expresiva, al experimentalismo lingüístico (y también narrativo) y a la carga ideológica de la obra de Consolo. Interesantes en especial artículos como el de Patrizia FARINELLI, "Strategie compositive, motivi e istanze nelle opere di Vincenzo Consolo», Italienisch, 37, 1997, p. 38-45; o Norma Bouchard, "Consolo, Lévinas and the Ethics of Posmodernist Storytelling", Annali d'Italianistica, 19, 2001, p. 119-136: la citada interrelación en la escritura de Consolo, lejos de resolverse en un relativismo ético (por la destrucción de los discursos normalmente dedicados a los grands récits de la modernidad) o en una pasiva resignación, siguiendo las directrices del pensamiento de Lévinas, encontraría su razón de ser, radicalmente comprometida, en este eje («As Consolo suggests [...] the reciprocity between ethics and novelistic discourse depends upon a narrative and linguistic experimentation", BOUCHARD, op. cit., p. 134); en el rechazo de una filosofía «ilusionística" acerca de la historia (es decir, el concepto de una sola historia) por el de una pluralidad de historias (o de una historia contingente): Ruth Glynn, «Metaphor and Philosophy of History: Motifs of Representation in Consolo's Sorriso dell'ignoto marinaio", Italian Studies, 54, 1999, p. 118-131 (aquí p. 119-121). Cfr. También Miguel G. OcHOA, «Historia, ficción y narración en la narrativa de Vincenzo Consolo", Espéculo: Revista de Estudios Literarios, 13, 1999-2000.

4. Vincenzo Consolo, «Lo spazio in letteratura», en ÍDEM, Di qua dal faro, Milán: Mondadori, 2001 [1999], p. 263-270 (aquí p. 265). La idea es, como cabría esperarse, recurrente en su ya mencionado Il Viaggio di Odisseo, op. cit. 
Nel viaggio di quell' Ignoto sulla traccia d'un triangolo che aveva per vertici Messina, Lipari, Cefalù, vide il viaggio d'ogni uomo, l'avventura d'ogni Ulisse: la fuga dal terremoto, dal disastro, dal mortale rischio dello Stretto, la sosta nell'incerto regno dei venti, delle tempeste, delle eruzioni dei vulcani, l'approdo infine in un paese di lunga e ferma storia, il rifugio nelle solide mura d'una casa. ${ }^{5}$

El viaje de regreso, el nostos, por otra parte, le permite a Consolo establecer su filiación de escritor siciliano, trazar el itinerario de su más inmediata tradición literaria de referencia, construido éste a partir de analogías percibidas en el modo de abordar el tema del regreso a la isla y en el modo cómo en este contexto se entretejen las referencias históricas extratextuales: en efecto, el inevitable alejamiento de la isla y el regreso, qué energías y consideraciones son puestas allí en juego, a qué tratamiento del espacio son asociados y cómo son planteados, se convierten en los ejes con que el autor moldea su lista de afinidades literarias. ${ }^{6}$ Desde el Verga del recinto de Acitrezza hasta el posicionamiento más fructífero para Consolo, el de Vittorini, a cuya «lección» se remitirá en repetidas ocasiones, pasando, en particular, por Pirandello y Sciascia. Se habla, por ejemplo, en I ritorni del arquetipo "universal» homérico de la insularidad para enlazar con el nostos y describir de esta forma la constelación de significados puesta en movimiento, en relación con Sicilia, para los escritores allí nacidos. Se va, en este contexto, de la isla salvaje de Homero a la que resulta de la superposición histórica de múltiples y heterogéneos estratos, responsables de esa singularidad precisa por la que de algún modo se vería incrementado lo insular, marginal o periférico de Sicilia: de donde la necesidad que los escritores sicilianos parecen sentir de emprender el viaje hacia centros «históricos, culturales, lingüísticos» desde los cuales más tarde volver. ${ }^{7}$ Este doble movimiento ineludible es una idea recurrente que se presenta en más de una ocasión de forma similar en los textos de Consolo. Desde una perspectiva global, más interesante resulta para nuestros propósitos constatar que no hay que esperarse en estos textos equivalencias simples en relación con el nostos, a cómo éste es presentado en ellos como elemento estructural de una concreta línea de la literatura siciliana y de su relación con la isla. Su uso no es unívoco, coexisten en el nostos consoliano aspectos de signo diferente, se ponen de manifiesto en él distintos motivos. Del regreso a la isla como recuperación del bien perdido y completamiento del viaje iniciático (lo que podríamos remitir a la imagen del regreso feliz a Itaca), al regreso como regresión (es decir, la caída en la cueva de Calipso), pasando por momentos de armónicos más arquetípicos (Sicilia como prueba iniciática, metonimia de Escila y Caribdis); en lo más concreto, el contraste con el hecho histórico actual (aquí el nostos sirve para poner en

5. Vincenzo Consolo, L’Olivo e l'olivastro, Milán: Mondadori, 1999 [1994], p. 124.

6. Cfr. Vincenzo COnSOlO, «I ritorni», en ÍDEM, Di qua dal faro, Milán: Mondadori, 2001 [1999], p. 138-145; "Lo spazio in letteratura», op. cit. (en especial p. 268-70); Il viaggio di Odisseo, op. cit., p. 13 s.

7. «I ritorni», op. cit., p. 138. 
relieve la distancia entre la devastación real e histórica en referencia al arquetipo de totalidad absoluta encarnada por el texto homérico). Para usar las imágenes reiteradas del autor al respecto: el olivo, la cueva maternal, el estrecho de Mesina como una suerte de línea de sombra, el olivastro, respectivamente. Todos ellos elementos que se alternan e incluso, en ocasiones, coinciden en la estructura del nostos consoliano.

Según Consolo, decíamos, es la parábola que se lee en Vittorini el lugar donde él mismo encontró la clave para una relación positiva con su propia insularidad. Sería ésta la «lección» vittoriniana: es posible un regreso que libre del desarraigo y que no repercuta en una actitud regresiva, sino que se materialice en gesto positivo de proyección:

Ecco, Vittorini indica, con il suo nostos, l'ambivalenza del mito: la necessità dello sprofondamento nelle viscere materne, nelle matrici della memoria e il dovere di risalire, di ripartire, di approdare alla terra dei padri, della società, della storia. Guai a rimanere isolati o esiliati nella sfera materna. E, del resto, quella di Silvestro-Vittorini non è una vecchia madre mediterranea che esilia il figlio, non è una Circe, una Calipso o una Didone, è una madre nuova, una nuova donna, consapevole, attiva, "progressiva». [...] Dopo questa lunga premessa [...] dichiaro la lezione appresa da Vittorini del viaggio, del movimento, e con essa l'idea di letteratura, che deriva naturalmente da una ideologia politica. ${ }^{8}$

Según esta idea, es posible hallar un camino de regreso que fructifique en un equilibrio de experiencias. El hecho es que en los textos de Vincenzo Consolo las referencias al mundo del olivo, a la idea de Itaca y el nostos como experiencia total que podría ligarse a tal objetivo, se encuentran contrastadas duramente por las tensiones materializadas en la terca realidad del devenir. En los textos, en su concepción del trabajo literario, propio y ajeno, en su visión política de Italia. Dice Consolo, hablando siempre de escritores sicilianos:

Nei libri che fin qui abbiamo esaminato, da Conversazione in Sicilia a Horcynus Orca, non è mai presupposta l'esistenza di una società [...]. In Sicilia, questo, priva da sempre di una società, in quest'Itaca desiderata, raggiunta e rifiutata. In altri luoghi di questo paese era presupposta la società, in Toscana, in Piemonte, in Lombardia. [...] Sappiamo che dopo l'uccisione dei Proci [...] il riconoscimento e il ricongiungimento di Ulisse e di Penelope avviene dopo la rivelazione del segreto: il loro talamo era costruito su un tronco di ulivo. Nel viaggio di ritorno degli scrittori siciliani manca questa conclusione, manca questo alto simbolo della civiltà. ${ }^{?}$

El correlato principal de tales tensiones es, en los textos de Consolo, un concepto muy característico y de sobras conocido: el binomio, que da título a su obra más emblemática al respecto, formado por el olivo silvestre o aze-

9. «I ritorni», op. cit., p. 145. 
buche, el olivastro, en oposición al olivo y los valores vehiculados por su imagen. Si la Odisea homérica se resuelve en un equilibrio entre los dos símbolos, con el olivo como centro, Consolo acepta la ausencia de éste como verdadera cifra de la existencia contemporánea:

Anche chi qui scrive, nella sua vicenda letteraria, attraverso la lezione vittoriana, è risalito, soprattutto nei due ultimi libri, L'olivo e l'olivastro, e Lo spasimo di Palermo, all'archetipo omerico, a quell'Odissea da cui siamo partiti. Lo spazio, in questi due romanzi, si dispiega fra due poli, Milano e la Sicilia. Ma l'Odissea, sappiamo, è una metafora della vita, del viaggio della vita. Casualmente nasciamo in un'Itaca dove tramiamo i nostri affetti, dove piantiamo i nostri olivi, dove attorno all'olivo costruiamo il nostro talamo nuziale, dove generiamo i nostri figli. Ecco, noi oggi, esuli, erranti, non aneliamo che a ritornare a Itaca, a ritrovare l'olivo. Lo scacco consiste nel fatto che sull'olivo ha prevalso l'olivastro, l'olivo selvatico. ${ }^{10}$

Otros motivos redundan en la constelación descrita. El tratamiento de Escila y Caribdis, por ejemplo, prueba iniciática de tintes arquetípicos:

Allora per noi diventa quel braccio di mare, quel fiume salmastro, il suo mito, la metafora dell'esistenza, lo stretto obbligato, il tormentato passaggio in cui l'uomo può perdersi, può perdere la ragione, imbestiandosi, o la vita contro lo scoglio o dentro il vortice di una natura maligna, mostruosa e feroce; o salvarsi, uscire dall'orrido caos, dopo il passaggio cruciale, e approdare, nell'Itaca della realtà e della storia, della ragione e degli affetti. ${ }^{11}$

Puede también revelarse de forma más concreta como correlato de una condición vital ligada intrínsecamente a Sicilia:

Una metafora anche diventa lo stretto di quel che serva la vita a un uomo nato per caso in Sicilia: epifania crudele, periglioso sbandare nella procella del mare, nell'infernale natura; salvezza infine possibile, dopo molto travaglio, approdo a un'amara saggezza, a una disillusa intelligenza. ${ }^{12}$

Se toca aquí por otra parte un elemento para nada secundario en la cuestión, es decir, el hecho de que Sicilia forma parte de la geografía real de los escenarios homéricos. Más allá de las discusiones por las localizaciones físicas de la Odisea, el incuestionable valor emblemático del espacio siciliano como referencia homérica conlleva, en las obras de Consolo, interesantes sobreposiciones del material simbólico — alejadas, repitamos, de una eventual coherencia

10. "Lo spazio in letteratura», op. cit., p. 270; palabras casi idénticas, en Il viaggio di Odisseo, op. cit., p. 23 s.

11. Vincenzo Consolo, "Vedute dallo stretto di Messina», en ÍDEM, Di qua dal faro, Milán: Mondadori, 2001 [1999], p. 67-91 (aquí p. 68) (cfr. palabras casi idénticas en L'olivo e l'olivastro p. 21-22).

12. Ibídem. 
unívoca-. Esto explica porqué Escila y Caribdis, que en efecto muy probablemente cabría situar en el estrecho de Mesina, pueden transferir su fuerza simbólica, su naturaleza de prueba iniciática a toda Sicilia y ser punto de partida de un itinerario circular, si atendemos a las diferentes acepciones posibles: Sicilia que se convierte, como vemos, en prueba que quien nace allí debe superar para poder regresar a su Itaca, naturalmente la misma Sicilia. A fin de cuentas, la enorme fuerza de las imágenes homéricas en el discurso de Consolo se cimienta sin duda sobre un ideal, perdido si se quiere, pero con vitalidad crucial — moral, se diría- La lección aquí, en este sentido, sería la de Goethe, sólo que corregida a la luz del contraste histórico. Si el poeta alemán podía ensimismarse aún en aquella estupefaciente realidad que le parecía la misma de Homero y ver en Sicilia el emblema de la plenitud esencial del mundo homérico, para Consolo la lección, la amara saggezza, se resume en el predominio del olivastro de que hablábamos, cuya materialización se encontraría en la continua denuncia por parte del autor de la Sicilia actual en términos de devastación, corrupción, abuso, crimen. En esta dimensión, adquiere relieve el nostos consoliano: el retorno es imposible, los pretendientes, los cíclopes, las fuerzas monstruosas, en general, han devastado el lugar de regreso; más bien, se diría: han profanado abyectamente el lugar del recuerdo. Nostos como conocimiento doloroso. ${ }^{13}$

Este tipo de conocimiento es en efecto tratado por el autor reiteradamente. Puede encontrarse en el contraste concreto entre lugares físicos reales, como sucede con el episodio de las vacas del sol, tradicionalmente ubicados en un lugar que el viajero de L'olivo e l'olivastro observa con horror:

Sulla piana dove pascolavano gli armenti del Sole, dove si coltivava il gelsomino, è sorta una vasta e fitta città di silos, di tralicci, di ciminiere che perennemente vomitano fiamme e fumo, una metallica, infernale città di Dite che tutto ha sconvolto e avvelenato: terra, cielo, mare, menti, cultura. ${ }^{14}$

13. «Ma importante per noi, oltre che provvista di una scala di valori, è la riflessione di Consolo sulle proprie opere e sul cammino in esse dell'idea di sradicamento, di viaggio verso la storia e di nuovo viaggio verso l'isola di origine, dove però la terra della memoria è scomparsa, cancellata da nuovi Proci come se fosse l'utopia vittoriniana a essere stata cancellata. A questo punto c'è lo stacco verso l'idea di un viaggio penitenziale e liberatorio di Odisseo. Le colpe non sono più soggettive, ma della storia: i mostri sono concreti reali; non vengono più dal mare, sono terrestri, vengono da un potere mafioso che rende la terra inabitabile» (Maria CORTI, "Introduzione», en Vincenzo CONSOlO, Mario NICOLAO, Il viaggio di Odisseo, Milán: Bompiani, 1999, p. V-VIII, aquí p. VII). Sólo gracias a la mediación lingüística se resolvería el imposible retorno, sobre la base de la triple articulación entre memoria, lengua y territorio, según Francesca NERI, Giampieri SEGNERI, «Reshaping Memory: Bufalino, Consolo and the Sicilian Tradition», European Studies, 18 (2002) p. 91-105: "The destruction brought about in these years concerned Sicilian society and culture, and started with an attack on the landscape. [...] Thus the horror of history leaves its imprint on the land, forever making impossible and idyllic or a-historical rendering of the landscape. [...] This paper will deal mainly with three themes: memory, language and territory. Their order is not random, as our starting hypothesis is that language creates for these writers the only possible mediation between history and geography, between the past and contemporary reality», p. 94-95.

14. L'Olivo e l'olivastro, op. cit., p. 28. 
O, en general, de forma más abstracta, en su ir y venir por la isla. Gela sería el emblema perfecto al respecto, esa Gela que el autor, en las páginas ya citadas de I ritorni, exponía como lugar de la mayor desilusión personal, prueba inapelable de ese imposible retorno al olivo tan distintivo de los escritores sicilianos. Gela es el lugar donde para Consolo la ausencia del olivo es más clamorosa: ${ }^{15}$

Dire di Gela nel modo più vero e forte, dire di questo estremo disumano, quest'olivastro, questo frutto amaro, questo feto osceno del potere e del progresso, dire del suo male infinite volte detto, dirlo fuor di racconto, di metafora, è impresa ardua o vana. [...] Da quei pozzi, da quelle ciminiere sopra templi e necropoli, da quei sottosuoli d'ammassi di madrepore e di ossa, di tufi scanalati, cocci dipinti, dall'acropoli sul colle difesa da muraglie, dalla spiaggia aperta a ogni sbarco, dal secco paese povero e obliato partì il terremoto, lo sconvolgimento, partì l'inferno d'oggi. Nacque la Gela repentina e nuova della separazione tra i tecnici, i geologi e i contabili giunti da Metanopoli [...] e gli indigeni dell'edilizia selvaggia e abusiva, delle case di mattoni e tondini lebbrosi in mezzo al fango e all'immondizia di quartieri incastati, di strade innominate, la Gela dal mare grasso d'oli, dai frangiglutti di cemento, dal porto di navi incagliate nei fondali, inclinate sopra un fianco, isole di ruggini, di plastiche e di ratti; nacque la Gela della perdita d'ogni memoria e senso, del gelo della mente e dell' afasia, del linguaggio turpe della siringa e del coltello, della marmitta fragorosa e del tritolo. ${ }^{16}$

Es en páginas inmediatamente anteriores a éstas donde se descubren las palabras más explícitas acerca de esta Ítaca perdida. El olivastro como objeto de una mise en abyme que proyecta su denuncia esencial sobre todo el presente:

Sembra così, questo piccolo paese [Caltagirone] nel cuore della Sicilia, il plastico, l'emblema del più grande paese, della vecchia Italia che ha generato dopo i disastri del fascismo, nei cinquant'anni di potere, il regime democristiano, la trista, alienata, feroce nuova Italia del massacro della memoria, dell'identità, della decenza e della civiltà, l'Italia corrotta, imbarbarita, del saccheggio, delle speculazioni, della mafia, delle stragi, della droga, delle macchine, del calcio, della televisione e delle lotterie, del chiasso e dei veleni. Il plastico dell'Italia che creerà altri orrori, altre mostruosità, altre ciclopiche demenze. ${ }^{17}$

En realidad, ya la Odisea, tal como la lee Consolo, podía aludir en su interior a la expiación de una culpa no muy distinta de la que se vislumbra en estas palabras. Los padecimientos de Ulises, el horror encarnado en Escila y Caribdis, los cíclopes, podrían ser vistos como un contrapaso, la condena por el artefacto diabólico de muerte, el caballo. Podría verse en éste, por otra parte, el 
emblema de la pregunta inquietante por la técnica y el hombre, por la realidad de la técnica aplicada a la violencia en el mundo actual. El mismo autor vuelve en diferentes ocasiones a estos conceptos:

Ci sembrano Scilla e Cariddi, nella loro moltiplicazione di mostruosità, nel loro acquattarsi, nell'occultare la loro ferocia [...], nella loro implacabile distruttività, quasi il contrappasso del mostro artificiale, bellico, del cavallo di legno ideato da Ulisse, di quell'unico corpo che nasconde nel buio suo ventre molteplici teste, molteplici braccia ferali. ${ }^{18}$

De esa conciencia de pecado, que implica en realidad al hombre en general, surge la necesidad imperiosa de una misión para la literatura:

Nella modernità, le colpe non sono più soggettive, ma oggettive, sono della storia. I mostri non sorgono più dal mare, dalla profondità del subconscio, ma sono mostri concreti, reali, che tutti noi abbiamo creato (tutti noi abbiamo scatenato le guerre, creato i campi di sterminio, le pulizie etniche, lasciamo morire per fame la stragrande maggioranza dell'umanità...). Nessun viaggio penitenziale e liberatorio è ormai possibile. Itaca non è più raggiungibile. Questo, secondo me, lo scrittore oggi ha il compito di dire, di narrare. ${ }^{19}$

Así pues, abundan las referencias que podrían ser de nuestro interés. Claro está, para Consolo no todo se reduce únicamente al nostos de la Odisea, pero el intertexto homérico es con mucho el más importante en su obra. Y, en realidad, su tratamiento en ella puede considerarse una suerte de tonalidad de referencia para otros elementos de naturaleza parecida, entre los que, como se decía en su momento, me parece interesante contemplar el laberinto, el segundo de los ámbitos temáticos que anunciábamos al empezar estas páginas. Destaco como punto de partida un paralelismo que surge en L'olivo e l'olivastro, entre una aparición del Minotauro y las ideas que hemos tratado hasta este momento. Como en el imposible nostos, como en el olivastro invasor, también en lo que se refiere al habitante monstruoso del centro del laberinto el texto de Consolo no se remite unívocamente a términos como liberación final, conocimiento, "centro" de experiencia. Ulises no puede volver o, si vuelve, encuentra Ítaca destruida; del mismo modo, no hay ningún Teseo que salve del reinar del monstruo:

Ritorna il toro a Cnosso, la bestia potente che irrompe sull'orlo di un fasto che si sfalda e decade, sforza e invade regine di noie e mollezze. Il prezzo di tanto regresso, il ritorno ad ere pregresse, è il sacrificio barbarico a fatali scadenze. Nessun Teseo qui giunge, nessun può liberar dall'oltraggio l'Atene civile. ${ }^{20}$

18. "Vedute dallo stretto di Messina», op. cit., p. 67, cfr. L'olivo e l'olivastro, op. cit., p. 19 s.

19. Il viaggio di Odisseo, op. cit., p. 22.

20. L'olivo e l'olivastro, op. cit., p. 147. 
El trazado paralelo es claro, aunque me gustaría ir más allá y referirme a otra obra más alejada en el tiempo, donde el tema del laberinto tenía un peso específico muy relevante, Il sorriso dell'ignoto marinaio, esa famosa novela que marcó la pauta por la que se distinguiría la literatura de Consolo, con la nítida silueta de su radical posicionamiento estilístico, narrativo e ideológico, y que ha ido asumiendo, para lectores, críticos y el mismo autor, la consideración de punto central de su obra. Dejando a parte la amalgama laberíntica de materiales de diferente factura, procedencia, perspectiva y estilo que componen el libro; sin entrar a discutir el intricado trazado narrativo de su desarrollo, el lector recordará la imagen, con que se cierra (casi) el libro, de la planta en espiral de la prisión visitada por el protagonista Mandralisca. Mucho se ha hablado de esta sugestiva referencia a la espiral como forma laberíntica, de su vínculo con la afición a la malacología del protagonista y su relación estrecha con la totalidad de la economía de la novela, sobretodo a partir de que Segre, en su penetrante introducción, elevara, uniendo lo político con lo estilístico, la estructura espiral a una estructura base con que interpretar la obra:

Questo capitolo è tutto attaversato dalla metafora della chiocciola, metafora plurima che designa successivamente i privilegi della cultura, l'ingiustizia del potere, la proprietà come usurpazione. La metafora realizza una sorta di autocritica, dato che di chiocciole si occupa principalmente Mandralisca nelle sue ricerche scientifiche; diventa poi schema descrittivo, nel capitolo ottavo, quando si parla del carcere di Sant'Agata di Militello [...] Lo schema elicoidale della chiocciola può servire bene per analizzare il romanzo, come ci autorizza a fare Consolo [...]. Come sono raffigurati i pensieri nel Sorriso dell'ignoto marinaio? V'è una inarrestabile discesa spiraliforme [...]. Questa discesa è anche linguistica: al sommo c'è il linguaggio vivido e barocco dei primi capitoli; negli inferi (nono e ultimo capitolo), le scritte compediarie dei prigionieri $[\ldots] .{ }^{21}$

El esquema, con imagen gráfica incluida, responderá, sin lugar a dudas, a la distribución de los escritos de los prisioneros y sus cambiantes características, lingüísticas ante todo. Pero es cierto que su efecto no se quedará allí: la crítica en efecto ha señalado la rica constelación que orbita en las curvas de esta espiral. En este sentido, hay que referirse al hecho de que la polivalencia típica de determinadas imágenes emblemáticas (la Luna, el azebuche, Rosalía, el cuadro de Antonello da Messina, etc.), el desvelamiento de cuyos significados puede reverberar en ecos de naturaleza estructural, es una característica de la narrativa consoliana. ${ }^{22}$ En primer lugar, sin duda alguna, se señalará la rela-

\section{C. SEGRE, op. cit., p. 80-81.}

22. "Caratteristico di simili immagini è il fatto di possedere, per convenzione o per intenzione dell'autore, polivalenza semantica [...]. Come la luna, appunto [...]. Nel Sorriso dell'ignoto marinaio e in Retablo (1987) determinati elementi emblematici acquistano una funzione anche per la struttura narrativa. È infatti nel graduale processo di disvelamento del significato di tali elementi che le storie trovano un filo conduttore» (P. FARINELLI, op. cit., p. 39). 
ción de la imagen con una estéril actividad intelectual, como emblema en cierto sentido de impostura. ${ }^{23}$ Pero cabe la posibilidad de pensar también su aparición como elemento especular de las líneas de tensión entre el ayer y el mañana presentes en el libro: tanto planimetría de una prisión como vehículo de un primer intento de dar la palabra a los marginados, tanto elemento científico como reflexión sobre el lenguaje. ${ }^{24} \mathrm{El}$ mismo Consolo describía así la parábola espacial contenida en la novela, y su significación:

I tre elementi allora, la rivolta contadina di Alcàra, i cavatori di pomice di Lipari e il Ritratto d'Antonello reclamavano una disposizione su uno spazio di rispondenze e di senso, in cui il Ritratto stesso, nel suo presumibile percorso da una Messina, già di forte conessione storica, cancellata dai terremoti, a Lipari, isola-regno d'esistenza, di mito, a Cefalù, aprodo nella storia e nella cultura, disegnava un triangolo e un movimento da un mare d'incertezza e rassegnato destino (l'Aci Trezza e le falde etnee verghiane) a una terra di consapevolezza e di dialettica. Questa planimetria metaforica verticalizzavo poi con un simbolo offertomi dal malacologo Mandralisca, quello della conchiglia, del suo movimento a spirale (archetipo biologico e origine di percezione, conoscenza e costruzione, com'è nella Spirale delle calviniane Cosmicomiche; arcaico segno centrifugo e centripeto di monocentrico labirinto, com'è in Kereny $\mathrm{e}$ in Eliade). ${ }^{25}$

A propósito de estas afirmaciones, se desprende del estudio de Traina la idea de una evolución dinámica en la misma autoconciencia autorial acerca de la imagen. Según el estudioso, en el momento de escribir Il sorriso dell'ignoto marinaio, el autor tomaba como referencia más bien a Calvino, mientras

23. «Se Interdonato è il modello dell'integerrimo rivoluzionario giacobino e inflessibile intellettuale engagé, Mandralisca rappresenta la difficile maturazione dell'erudito di provincia che riesce a passare, alla fine del romanzo, da un generico riformismo liberale a un'autentica e solidale comprensione delle esigenze popolari. Metafora di questo passaggio è la discesa nel carcere a forma elicoidale, labirintica chiocciola ben più viva e reale delle chiocciole o conchiglie o lumache studiate fin allora con passione di malacologo» (G. TRAINA, op. cit., p. 20; cfr. también, Ibid., p. 60 s.).

24. "The chiocciola provides the structure for both the prison building and the narration which Mandralisca suggests is the first attempt at enabling the underprivileged to write their own history. The snail metaphor also functions as a cohesive agent for all of Mandralisca's interests, both those of the past (art and study) and that which now occupies the politically committed Mandralisca - the role played by language and other forms of representation in the construction of reality and power» (R. GLYNN, op. cit., p. 129). La conversión de Mandralisca se vería reflejada en la espiral (Ibid., p. 130), pero tal reflejo podría proyectarse también sobre la esfera dominante en tal conversión, la lingüística: «The linguistic sophistication is accompanied by a variety of voices and of points of view, creating a rhetorically articulated prose, full of contrasts. An example would be the way Consolo uses the chiocciola [...] as a metaphor for pointless rational thinking, for the nobleman's vacuous passion for collecting things, for his inability to understand anything real. Furthermore, it can be used as a topological metaphor for Consolo's writing, not only as it appears in Il sorriso, but also in Retablo, in Nottetempo and in parts of the short stories» (F. NERI, G. SEGNERI, op. cit., p. 100).

25. Vincenzo CONSOLO, «Il Sorriso, vent’anni dopo», en ÍDEM, Di qua dal faro, Milán: Mondadori, 2001 [1999], p. 276-282 (aquí p. 278). 
que, cuando vuelve a publicarse el libro en 1987, con el texto crítico de Segre que subrayaba la centralidad de la imagen en espiral como introducción, nuevos significados habían engrosado el espesor de la metáfora para el mismo Consolo, significados a los que el escritor habría podido llegar a través de las traducciones en italiano, en los primeros ochenta, de los textos de Eliade y Kereny. ${ }^{26}$ Sea como fuere, la presencia de la espiral se basta por sí sola para poner en funcionamiento en su giro vertiginoso toda la carga sugestiva de su arcaico contenido. Es por ello que el laberinto puede convertirse, en el mismo estudio de Traina, en un fecundo elemento de análisis, en especial al trazar alternativas entre historia y naturaleza, el necesario viaje hacia el vientre materno, hacia las vísceras y el peligro de quedar prisioneros en ese magma. ${ }^{27}$

Ya Consolo nos indicaba el camino que tomar, no en su texto de presentación, sino ya en la misma novela, al citar explícitamente, en la descripción de la cárcel, a Còcalo, el rey de Sicilia al que la tradición liga un momento de la historia del laberinto (en concreto, no en la forma más elaborada de éste, sino precisamente en la espiral, que puede verse como su antecesora y que en realidad algunos autores prefieren no considerar aún laberinto): ${ }^{28}$

E siamo persuasi che quell'insolito e capriccioso nome chiuso tra le parentesi che vien dopo Girolamo del principe e del marchese, Còcalo, [...] abbia ispirato l'architetto. Essendo Còcalo il re di Sicilia che accolse Dedalo, il costruttore del Labirinto, dopo la fuga per il cielo da Creta e da Minosse, ed avendo il nome Còcalo dentro la radice l'idea della chiocciola, kochlías nella greca lingua, còchlea nella latina, enigma soluto, falso labirinto, con inizio e fine, chiara la bocca e scuro il fondo chiuso [...].

Cabría notar que «falso laberinto» la espiral lo es más que nada por su no cambiar sistemáticamente de dirección, y no por una posible característica naturaleza laberíntica, como parece al contrario desprenderse de estas palabras, puesto que en realidad, tampoco en el laberinto clásico se da la posibilidad de pérdida. El laberinto es más bien símbolo de un itinerario, de un recorrido iniciático que conlleva el máximo esfuerzo y el máximo recorrido, entre un punto y un centro, que precisa de cambios constantes de dirección y en alternancia de alejamiento y acercamiento a este centro. ${ }^{29}$ Es en este ámbito simbólico que la espiral, la espiral de Il sorriso dell'ignoto marinaio incluida, adquiere silueta de laberinto, tal como desarrollan in extenso las páginas citadas de Traina:

Il fatto è che quel castello [...] non possiede scale o scaloni in verticale, linee ritte, spigoli, angoli o quadrati, tutto si svolge in cerchio, in volute, in seni e

28. Hermann KeRn, Labirinti, Milán: Feltrinelli, 1981; Gioachino CHIARINI, Odisseo: Il labirinto marino, Roma: Kepos, 1992.

29. KERN, op. cit., p. 11-14; CHIARINI, op. cit., p. 15-19. 
avvolgimenti [...]. E la fantasia più fantastica di tutte si trova dispiegata in quel catojo profondo, ipogeo, sènia, imbuto torto, solfara a giravolta, che fa quasi da specchio, da faccia arrovesciata del corpo principale del castello sotto cui si spiega, il carcere: immensa chiocciola con la bocca in alto e l'apice in fondo, nel bujo e putridume.

La espiral, en abstracto, la concha, en concreto (como imagen de una afición alienante, o almenos estéril en su nula relación con la realidad histórica), se despliega pues en figura laberíntica. En ese espacio de regresión al infinito, Mandralisca, de alguna forma, supera un itinerario iniciático. La espiral plantearía en abyme el trayecto de todo el libro, con ese final que nos presenta un Mandralisca renacido para la historia, un hombre consciente de los crímenes del poder y comprometido ante los acontecimientos, que abandona su torre de marfil (en el fondo, un espacio infantil) para visitar el horror magmático y salir otro. La lección de Consolo, transmitir en la polifonía su apuesta contra el poder en su definición más capilar, se concreta aquí en un símbolo de arcaica resonancia. Aun así, la lectura del libro conlleva una ulterior complejidad, útil para evitar esquematismos simplistas, puesto que en realidad la narración no termina con esta imagen iniciática. Mandralisca no acaba renacido para un centro concreto, sino, como corresponde a un entorno tan polifónico y poliédrico, a una conciencia de vivir en un mundo sin centros. Los apéndices a modo de epílogos que complementan la obra parecen lanzar un mensaje pesimista, insinuando el predominio del poder, por refinadas que sean sus manifestaciones. La historia inclemente, después de una explosión de esperanza, está ya traicionando a ésta. En una visión en apariencia próxima a la del Gattopardo, pero en perspectiva diametralmente opuesta, ${ }^{30}$ estos apéndices transmiten visiones que contrastan con la perspectiva de un cambio positivo en referencia a las víctimas de las variadas formas del poder. Podríamos ver, en estos cáusticos apéndices, su oposición a la espiral iniciática, el anuncio de algo muy parecido a la idea que tanto hemos encontrado en Consolo: la certeza de que en el fondo va a prevalecer el olivastro. Y así, este laberinto puede reflejar un proceso esencial del individuo, pero su proyección va más allá, porque a la salida, el héroe puede comprobar que su despertar a la justicia ha sido un hecho aislado, que su recorrido no significa un punto final de redención personal y colectiva, sino un punto de partida en medio de ruinas. Empezar, sí, pero a partir de una conciencia de devastación.

Conciencia muy parecida, desde este punto de vista, a mi parecer, al nostos imposible del que antes nos hemos ocupado. El acercamiento no es gratuito. No estará de más recordar que sería posible afirmar, como demostró Chiarini, que el nostos de la Odisea y el laberinto clásico tienen mucho que ver uno con otro. En realidad, sostenía el estudioso en un sugestivo trabajo, ${ }^{31}$

30. Cfr. V. Consolo, "Il Sorriso, vent'anni dopo», op. cit., p. 279. Cfr. también G. Traina, op. cit., p. 18-19.

31. ChIARINI, op. cit. (la publicación es de 1991, pero elaboraciones previas del material datan de 1985). 
la forma laberinto (una de cuyas más primitivas versiones se remitía a la espiral, con Cócalo como personaje clave) servía para dar razón de la discutidísima lógica y emplazamiento de los viajes de Ulises. Más que referirse a una eventual geografía concreta de la Odisea, sobre cuya autenticidad no sería en definitiva necesario discurrir (cosa por otra parte previsible a la luz de los sucesivos intentos que este acercamiento crítico al texto homérico ha generado a lo largo de la historia), Chiarini sostenía que la clave podía ser atender a los movimientos trazados por los viajes de Ulises, en base a puntos de referencia cardinales; movimientos que cabría plasmar en las coordenadas hacia el este o hacia el oeste, hacia el centro o hacia la periferia, en paralelismo perfecto con el esquema de movimientos propuesto por el laberinto, esa danza mágica enigmáticamente presente en el escudo de Aquiles de la Iliada, donde se plasma de hecho la totalidad del cosmos. En otras palabras, el nostos de la Odisea reproduciría simbólicamente en el espacio literario la tipología espacial de movimientos del laberinto clásico. A formas paralelas, añadiríamos como corolario, cargas simbólicas similares. Se me permita subrayar que no se sostiene aquí una derivación concreta en el texto de Consolo. Manifiesto sólo una identidad en la longitud de onda de los motivos en cuestión. Nada hay de comprobable, pero a fin de cuentas no se trata de probar nada; nos encontramos ante dos ámbitos temáticos con semejanzas incuestionables: como fuente, como eco arquetípico, como uso renovado de ellos en el autor contemporáneo y como funcionalidad que adquieren en su obra. Es ahí donde se revela a mi entender muy fructífera la referencia a Chiarini como complemento a las líneas ya marcadas por Traina cuando comenta la aparición del laberinto en $\mathrm{Il}$ sorriso dell'ignoto marinaio. De hecho, el mismo Consolo en el libro entrevista, dejaba entrever lo cerca que estaban los dos elementos al presentar el movimiento de $I l$ sorriso en relación con el movimiento del viaje del nostos y con su concreción simbólica en la cárcel en espiral (en palabras casi idénticas a las citadas anteriormente):

Nel mio secondo e più conosciuto romanzo, Il sorriso dell'ignoto marinaio, il viaggio, il movimento è dal mare alla terra (all'inizio di ogni capitolo c'è sempre una nave che approda). Vuole essere quello il passaggio da un mare d'incertezza e di rassegnato destino (il mare di Acitrezza) alla terra della consapevolezza, della storia. Verticalizzavo poi il movimento con il simbolo offertomi dal malacologo Mandralisca, il protagonista del romanzo, il simbolo della conchiglia, del suo movimento a spirale (archetipo biologico e origine di percezione, conoscenza e costruzione, com’è nella Spirale delle calviniane Cosmicomiche [etc.]). ${ }^{32}$

Nostos y laberinto compartirían así un contenido idéntico, una misma base de danza cósmica; serían reflejos especulares de una misma tensión arquetípica: itinerario que es prueba iniciática, un esforzado camino de llegada a un 
centro, a una Ítaca. A una conjunción de olivo y olivastro, estaríamos tentados de decir parafraseando a Consolo. Sólo que ya sabemos que el nostos consoliano no nos devuelve a ningún centro, de la misma forma que tampoco el laberinto de $I l$ sorriso, a pesar de mantener en la esfera personal toda su dimensión de renacimiento, no se resuelve en un movimiento de redención colectivo, una katharsis. La historia que se intuye a través de todo ese relato, en realidad, tiene de positivo sólo la toma de conciencia del individuo y la toma de partido de éste por la justicia; su materializarse posterior llevará a ese laberinto insensato donde reina el Minotauro e inútilmente se esperará a un Teseo para realizar el recorrido. Inútilmente se volverá a esa Ítaca devastada e inalcanzable. El olivastro se habrá adueñado del territorio.

La pregunta es qué contar, cómo plantearse esta misión narrativa si el nostos, matriz de todo relato, no es ya posible, si el olivastro se ha extendido por doquier:

Alla base della nostra odissea moderna credo che ci sia solo l'olivastro, l'olivo selvatico: tempeste e naufragi, inganni o oblii, mutazioni, regressioni, perdite. C'è il ritorno del barbarico e mostruoso mondo dei Ciclopi o dei pirandelliani Giganti della montagna [...]. Ecco, nell'odissea moderna è avvenuta la separazione tra il selvatico e il coltivato. L'olivastro ha invaso il campo. Ulisse non può più seppellirsi sotto le sue foglie, dormire, morire e rinascere. Raggiunta Itaca, si accorge che l'isola è ormai distrutta, che lì ormai né Penelope né Telemaco lo attendono. ${ }^{33}$

Porque una coda parece importante añadir a estas reflexiones. El profundo sentido de pérdida, de extravío, característico del pensamiento y de la narración de Consolo encuentran correlativo simbólico en los motivos del nostos y el laberinto, que además se convierten en realidad en estructura que se refleja en el espejo de la construcción literaria. El nostos es la matriz de todo relato, ha afirmado repetidamente el autor. La imposibilidad de llevarlo a cabo no puede quedar sin implicaciones en la página escrita:

Ma voglio spostare il discorso su un altro piano, su quello della scomparsa nel nostro contesto, nella nostra civiltà di massa, dello schema omerico, del nostos. La scomparsa di questa matrice ha mutato il racconto, il romanzo, l'ha privato della memoria, lo ha chiuso in schemi, in generi ripetuti all'infinito, consumati, come il genere poliziesco, ad esempio. Il romanzo d'indagine poliziesca, con la sua semplice dinamica [...] acquieta illusoriamente la nostra ansia, la paura del vivere in questa nostra civiltà. ${ }^{34}$

Notemos las palabras con que describe el movimiento en Nottetempo, que pueden sintetizar las líneas de reflexión de todo nuestro artículo: 
Dall'esilio - l'esilio oggi dello scrittore in questa nostra civiltà di massa Marano-Consolo prende coscienza che la sua Isola, la sua Itaca, la terra della memoria è scomparsa, è stata devastata, cancellata dai Proci, che tutte le terre della memoria sono ormai ridotte a rovine. L'eroe sconfitto può compiere allora il viaggio solo per esprimere furore e dolore, piangere sulle rovine, Itaca insomma è diventata Troia, e nessun Enea, nessun Astianatte uscirà fuori dalle mura di quella città in fiamme. Voglio dire che l'utopia vittoriniana, l'utopia di Conversazione, de Le donne di Messina, de Le città del mondo è ormai irrimediabilmente crollata. È per questo che da Vittorini sono risalito a Omero, all'Odissea. Avevo bisogno di attingere a questa grande matrice del racconto per capire e per capirmi. ${ }^{35}$

En su elaboración por parte de un autor contemporáneo, nostos y laberinto conservan su fuerza simbólica. Si bien con toda probabilidad el origen de la espiral en Il sorriso dell'ignoto marinaio habría que buscarlo en la distribución de escritos y sus autores respectivos, y admitiendo que la reflexión consoliana acerca del laberinto, como describe Traina, podría además responder a dos momentos de conciencia distintos acerca del símbolo, es indudable que en la espiral concha, el eco del laberinto marino de Odiseo se hace notar con fuerza. El itinerario que lleva Mandralisca desde el regazo oscuro a la historia: aunque los epílogos nos muestren ya las fuerzas monstruosas al acecho; aunque, en el fondo, partiendo de esta situación de regresión, el laberinto no pueda llevar a ninguna otra parte que a la conciencia de destrucción que encarna, a la necesaria implicación ideológica y política de la conciencia del hombre ante el ultraje del poder. La misma amalgama de materiales literarios, esa famosa dificultad del estilo de Consolo, podrían ser un reflejo de esta errancia fundamental. El mismo rechazo de la forma novelesca tal como se concibe en general, se podrían considerar materializaciones de un mismo impulso. ${ }^{36}$ El nostos es imposible, el relato también. En más de una ocasión, en sus textos Consolo ha reclamado para sí las reflexiones hechas por Benjamin acerca de la narración. ${ }^{37}$ Se trata de un hilo que a mi entender sería fundamental abordar con mayor detención, pero que por de pronto nos permite una hipótesis con que cerrar aquí. Jamás, decía el filósofo alemán en el texto en cuestión, experiencias del calibre de la Primera Guerra Mundial habían merecido tal silencio por parte de sus protagonistas. ${ }^{38}$ Casi un siglo más tarde, Consolo hace suyas estas reflexiones desde una perspectiva más general: la historia contemporánea ha destruido las condiciones para la narración. La épica ha desaparecido, igualmente los narradores; la mayor losa, en la vida actual, es la del silencio.

36. "I ritorni», op. cit., p. 144; cfr. el comentario de G. TraINA, op. cit., p. 38-39 (incluida la referencia en nota a las reflexiones de A. Scuderi).

37. E. PAPA, op. cit., p. 191-192.

38. Walter BENJAMIN, «Il narratore. Considerazioni sull'opera di Nicola Leskov», en ÍDEM, Angelus Novus, Turín: Einaudi, 1995 [1962], p. 247-274. 
Tal es el espacio de donde la novela no puede surgir, de donde es necesario explorar nuevas vías expresivas que puedan superar la afasia estupefacta característica del hombre posmoderno. Se encuentra en este punto el lugar de donde parte el relato de Consolo. Un intento audaz de ir más allá del impasse. Retornos imposibles, laberintos rotos, olivastros invadiendo el paisaje. Es el «ahora no puede narrar»:

Ora non può narrare. Quanto preme e travaglia arresta il tempo, il labbro, spinge contro il muro alto, nel cerchio breve, scioglie il lamento, il pianto.

Es el relato concebido como strazio:

Una barca di pietra, la pietra in cui si mutò la barca feacica che aveva portato in patria l'eroe punito, l'eroe assolto dopo il lungo racconto — che in pietra si muti la barca, si saldi al fondale prima d'ogni ritorno, poiché nel ritorno, così nel racconto, consiste lo strazio. ${ }^{39}$ 